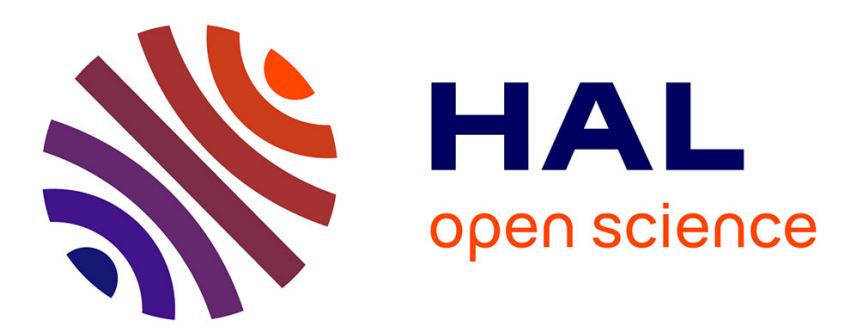

\title{
Calculation of the critical delay for the double inverted pendulum
}

Csenge A Molnar, Tamas Balogh, Islam Boussaada, Tamás Insperger

\section{To cite this version:}

Csenge A Molnar, Tamas Balogh, Islam Boussaada, Tamás Insperger. Calculation of the critical delay for the double inverted pendulum. Journal of Vibration and Control, 2020, 27 (3-4), pp.1 - 9. 10.1177/1077546320926909 . hal-02889890

\section{HAL Id: hal-02889890 https://hal.science/hal-02889890}

Submitted on 11 Feb 2021

HAL is a multi-disciplinary open access archive for the deposit and dissemination of scientific research documents, whether they are published or not. The documents may come from teaching and research institutions in France or abroad, or from public or private research centers.
L'archive ouverte pluridisciplinaire HAL, est destinée au dépôt et à la diffusion de documents scientifiques de niveau recherche, publiés ou non, émanant des établissements d'enseignement et de recherche français ou étrangers, des laboratoires publics ou privés. 


\title{
Calculation of the critical delay for the double inverted pendulum
}

\author{
Csenge A. Molnar \\ Department of Applied Mechanics, Budapest University of Technology and Economics and MTA-BME \\ Lendulet Human Balancing Research Group, Budapest, Hungary \\ Tamas Balogh \\ Department of Applied Mechanics, Budapest University of Technology and Economics and MTA-BME \\ Lendulet Human Balancing Research Group, Budapest, Hungary \\ Islam Boussaada \\ Université Paris-Saclay, Laboratoire des Signaux et Systèmes, CNRS-CentraleSupélec, Inria \\ Saclay-Île-de-France, 91192 Gif-sur-Yvette cedex, France \\ IPSA Ivry sur Seine, France \\ Tamas Insperger \\ Department of Applied Mechanics, Budapest University of Technology and Economics and MTA-BME \\ Lendulet Human Balancing Research Group, Budapest, Hungary
}

\begin{abstract}
Single and double inverted pendulum systems subjected to delayed state feedback are analyzed in terms of stabilizability. The maximum (critical) delay that allows a stable closed-loop system is determined via the Multiplicity-Induced-Dominancy (MID) property of the characteristic roots, i.e., the dominant (rightmost) roots are associated with higher multiplicity under certain conditions of the system parameters. Other methods, such as tracking the changes of the D-curves with increasing delay and the WaltonMarshall method are also demonstrated for the example of the single pendulum. For the double inverted pendulum subjected to full state feedback, the number of control gains is four, and application of numerical methods requires therefore high computational effort (i.e., optimization in a four-dimensional space). It is shown that, with the MID-
\end{abstract}

Email addresses: csenge.molnar@mm.bme.hu (Csenge A. Molnar),

tamas.balogh@mm.bme. hu (Tamas Balogh), Islam. Boussaada@l2s. centralesupelec.fr ( Islam Boussaada), insperger@mm. bme. hu (Tamas Insperger) 
based approach, the critical delay and the associated control gains can be determined directly using the characteristic equation and its derivatives.

Keywords: Delayed state feedback, stability, stabilizability, critical delay

\section{Introduction}

Stabilization of time delay systems is an important task in engineering control technology. Time delays typically arise in feedback systems and are often associated with unstable behaviour. Selection of the control parameters in order to stabilize the closedloop system is therefore a crucial task. Stabilizability is however limited by the size of the feedback delay [17, 34]. The critical delay, $\tau_{\text {crit }}$, is the maximum delay in the feedback loop, for which stabilization is still possible. If the delay is less than $\tau_{\text {crit }}$ then there exists a collection of control gains for which the closed-loop system is stable. If the delay is larger than $\tau_{\text {crit }}$ then the closed-loop system is unstable for any control gain combinations.

It is known that in some simple case studies, the limit of stabilizability and the critical delay are associated with multiple rightmost roots [13, 27, 4]. The so-called Multiplicity-Induced-Dominancy (MID) has proved to be a useful property in determining the critical delay for various classes of delay systems. Application of the MID property to scalar and to second-order equations subjected to delayed proportional feedback was conducted in [5] and in [6, 8], respectively. Extension to the delayed proportional-derivative (PD) feedback was studied in [4, 7] where dominancy is proven using the argument principle. Motivated by the above examples, critical delay of feedback systems may be determined by the analysis of the multiplicity of the rightmost characteristic roots.

In this paper, the MID-based method is applied to a two-degree-of-freedom undamped unstable system, namely, the double inverted pendulum, subjected to delayed PD feedback. First, the method is demonstrated for the benchmark problem of the inverted pendulum with delayed PD feedback, where a closed-form expression of the critical delay is established. Then, the critical delay is determined for the double inverted pendulum with two system parameters (length of the pendulums) and four con- 
trol gains (associated with the angular position and angular velocity of both pendulum segments).

\section{Stabilizability as function of feedback delay}

There are several analytical and numerical methods that analyze the stability and stabilizability of time delay systems. These allow to determine the regions of control gains and feedback delay for which the system may be stabilized. In the following subsections, two particular methods are briefly presented in the framework of the characteristic equation

$$
D(s, \tau)=A(s)+B(s) \mathrm{e}^{-s \tau}=0,
$$

where $\operatorname{deg}(A)>\operatorname{deg}(B)$. In the case of a mechanical system subjected to delayed feedback control, polynomial $A$ expresses the behavior of the plant, while $B$ corresponds to the controller. Indeed, the coefficients of $A$ typically represent mechanical properties of the system (mass, mass moment of inertia, center of gravity), and, therefore are considered to be fixed, while the coefficients of $B$ are associated with the control parameters to be tuned (control gains). The goal is to determine the values of the delay $\tau$ for which (1) has all its roots in the left-half plane.

\subsection{Walton-Marshall method}

The Walton-Marshall (WM) method [32] is a numerical tool that approximates the critical delay for fixed control and system parameters. The main steps of the method are the following. First, stability is determined for $\tau=0$. Then, the domain of the delay for which the system remains stable is established. If a root $s=\mathrm{i} \omega$ satisfies $D(s, \tau)=0$ for a certain $\tau$, then $D(\bar{s}, \tau)=0$ is also satisfied $(\bar{s}=-\mathrm{i} \omega$ is the complex conjugate of $s$ ). Consequently, the problem of finding roots on the imaginary axis reduces to solving the system of equations

$$
\left\{\begin{array}{l}
A(s)+B(s) \mathrm{e}^{-s \tau}=0 \\
A(-s)+B(-s) \mathrm{e}^{+s \tau}=0
\end{array} .\right.
$$

Eliminating the exponential term yields

$$
A(s) A(-s)-B(s) B(-s)=0 .
$$


Substituting $s=\mathrm{i} \omega$ into (3), one obtains a polynomial in $\omega^{2}$ :

$$
W\left(\omega^{2}\right)=A(\mathrm{i} \omega) A(-\mathrm{i} \omega)-B(\mathrm{i} \omega) B(-\mathrm{i} \omega)=0
$$

Imaginary roots correspond to positive $\omega^{2}$ solutions of (4). If there are no such roots, then the system is stable/unstable independently of the delay. Otherwise, candidates for the critical delay are obtained after substituting the corresponding root $s=\mathrm{i} \omega$ into (1), namely

$$
\tan \omega \tau=\frac{\operatorname{Im}\left\{\frac{A(\mathrm{i} \omega)}{B(\mathrm{i} \omega)}\right\}}{\operatorname{Re}\left\{\frac{-A(\mathrm{i} \omega)}{B(\mathrm{i} \omega)}\right\}} .
$$

The next move consists in investigating whether the corresponding root $s=\mathrm{i} \omega$ crosses the imaginary axis from left to right as $\tau$ increases. Indeed, in [32], the root crossing direction is determined by the properties of $W$. If a root $\omega$ crosses from left to right, then $W$ changes sign from positive to negative, i.e., $W^{\prime}\left(\omega^{2}\right)<0$. The maximum delay is determined by investigating all the crossing roots and their corresponding crossing directions.

\subsection{Multiplicity-Induced-Dominancy}

It is shown in [8, 7], that the admissible multiplicity of the zero spectral value for (1) is bounded by the Polya and Szegó bound denoted by $P B_{S}$, which is the degree of the quasipolynomial (i.e., the sum of the polynomials' degrees plus the number of delays). The critical delay and the corresponding critical control gains are associated with the rightmost root $s=0$ achieving the maximal admissible multiplicity. While multiplicity can be investigated by the vanishing of the successive derivatives of (1) with respect to $s$, the dominancy of the root $s=0$ requires further inquiry in most cases. This latter property is demonstrated in the next sections for both the single and the double inverted pendulum.

\section{Motivation: single inverted pendulum}

Stabilization of an inverted pendulum with delayed feedback is a benchmark problem in control engineering literature [2, 28, 26, 33] as well as in understanding human 
balancing and human motor control [18, 21, 20, 23]. As a matter of fact, many control concepts are often implemented in simple inverted pendulum systems, see, e.g., [22, 12, 26, 11]. As mentioned in the Introduction, stabilization by a delayed state feedback is not possible if the delay is larger than a critical value [30]. In this section, the stabilization of the single inverted pendulum is presented briefly, and the relation between the multiplicity of the rightmost characteristic roots and the critical delay is demonstrated. The critical delay is determined by three different techniques: 1) by analytical derivations; 2) by the MID approach; and 3) by the WM method.

\subsection{Mechanical model}

The mechanical model of the pendulum-cart system is shown in Fig. 1. The mass of the cart is assumed to be negligible compared to the mass of the pendulum. The linearized equation governing the motion of the stick is

$$
\ddot{\varphi}(t)-\frac{m g l}{2 I} \varphi(t)=\frac{l}{2 I} F(t),
$$

where $\varphi$ is the angular position of the stick, $m, l$ and $I=1 / 12 m l^{2}$ are the mass, length and the mass moment of inertia of the stick, respectively, $g$ is the gravitational acceleration and $F$ is the control force. In case of delayed PD feedback of the angular position, the control force may be modeled as

$$
F(t)=k_{\mathrm{p}} \varphi(t-\tau)+k_{\mathrm{d}} \dot{\varphi}(t-\tau),
$$

where $k_{\mathrm{p}}$ and $k_{\mathrm{d}}$ are the proportional and the derivative control gains, respectively, and $\tau$ is the feedback delay.

Hence, the characteristic function generated by (6) and (7) reads

$$
D(s)=s^{2}+a_{0}+\left(b_{0}+b_{1} s\right) \mathrm{e}^{-s \tau},
$$

where

$$
a_{0}=-\frac{m g l}{2 I}, \quad b_{0}=\frac{k_{\mathrm{p}} l}{2 I}, \quad b_{1}=\frac{k_{\mathrm{d}} l}{2 I} .
$$

It can be seen that the coefficient $a_{0}$ involves only the plant parameters $(m, I, l)$, while $b_{0}$ and $b_{1}$ depend on the control parameters $k_{\mathrm{p}}$ and $k_{\mathrm{d}}$, too. 


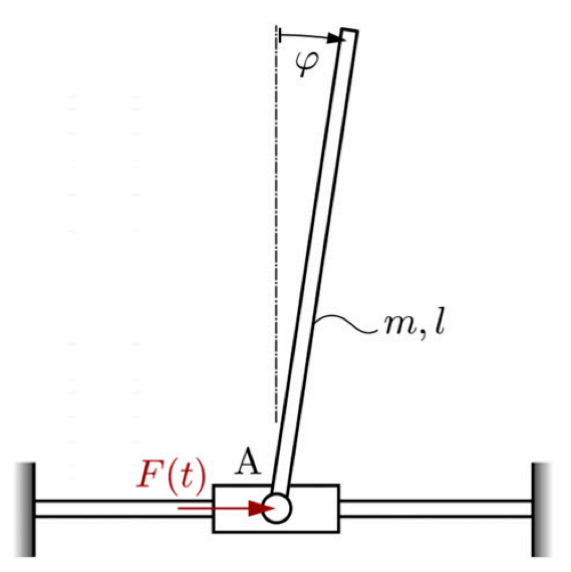

Figure 1: Mechanical model of the pendulum-cart system.

\subsection{Analytical stabilizability analysis}

Substituting $s= \pm \mathrm{i} \omega(\omega \geq 0)$ into the characteristic equation 8 and decomposing into real and imaginary parts yields the D-curves in the implicit form

$$
\begin{array}{r}
a_{0}-\omega^{2}+b_{0} \cos (\tau \omega)+b_{1} \omega \sin (\tau \omega)=0, \\
b_{1} \omega \cos (\tau \omega)-b_{0} \sin (\tau \omega)=0 .
\end{array}
$$

If $\omega=0$ then 10 gives $b_{0}=-a_{0}$, which is a so-called real root boundary [1]. If $\omega>0$, then (10) and (11) give the parametric curve

$$
\begin{aligned}
& b_{0}=\left(\omega^{2}-a_{0}\right) \cos (\omega \tau), \\
& b_{1}=\frac{\omega^{2}-a_{0}}{\omega} \sin (\omega \tau),
\end{aligned}
$$

which is called complex root boundary [1]. The D-curves split the plane $\left(b_{0}, b_{1}\right)$ into an infinite number of domains, where each domain exhibits a constant number of unstable characteristic roots. Stability can be attained by computing the number of unstable 

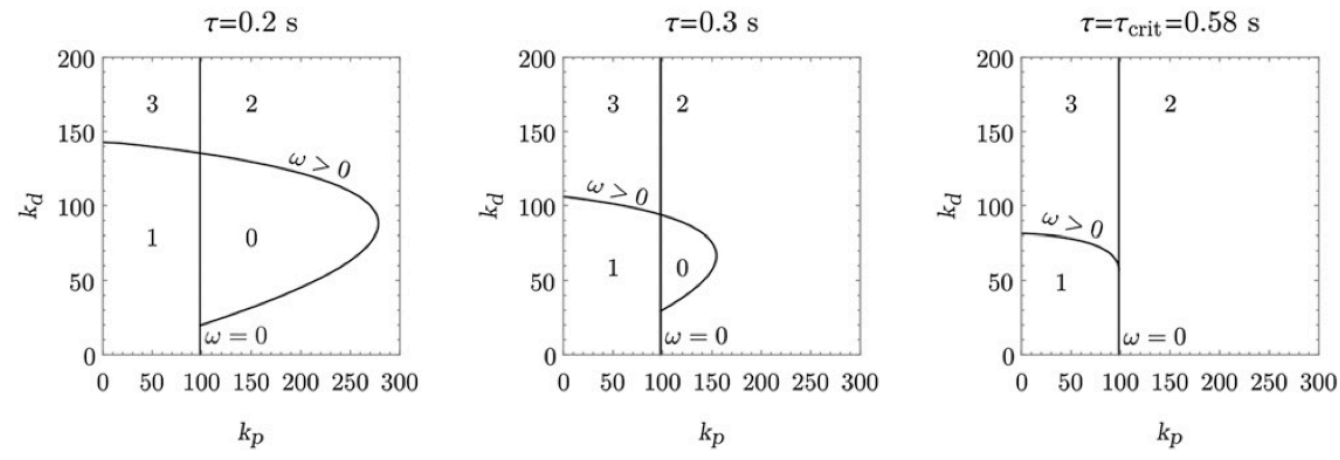

Figure 2: D-curves and the number of unstable characteristic roots of 8 for different delays. The stable region disappears when $\tau=\tau_{\text {crit }}$ and stabilization is not possible if $\tau>\tau_{\text {crit }}$.

characteristic roots in each individual domain and/or by checking the root tendency (root-crossing direction) along the D-curves [29, 24, 19]. Some sample stability diagrams for different feedback delays are depicted in Fig. 2 for the set of parameters in Table 1

As observed in Fig. 2, the stable domain shrinks with increasing feedback delay, and completely vanished at a critical value $\tau_{\text {crit }}$, when the tangent of the parametric curve 37 - 38 is vertical. This happens at the limit

$$
\lim _{\omega \rightarrow 0} \frac{\mathrm{d} k_{\mathrm{p}}}{\mathrm{d} k_{\mathrm{d}}}=\lim _{\omega \rightarrow 0} \frac{\frac{\mathrm{d} k_{\mathrm{p}}}{\mathrm{d} \omega}}{\frac{\mathrm{d} k_{\mathrm{d}}}{\mathrm{d} \omega}}=\frac{3 a_{0} \tau^{2}+6}{a_{0} \tau^{3}+6 \tau}=0,
$$

from which we infer, the critical delay $\tau_{\text {crit }}=\sqrt{-2 / a_{0}}$, i.e., $\tau_{\text {crit }}=0.58 \mathrm{~s}$ for the set of parameters in Table 1 . 


\begin{tabular}{ccc}
\multicolumn{3}{c}{ Table 1: Mechanical parameters of the inverted single pendulum } \\
\hline Parameter & Notation & Value \\
\hline Mass & $m$ & $10 \mathrm{~kg}$ \\
Length & $l$ & $10 \mathrm{~m}$ \\
Mass moment of inertia & $I$ & $83.33 \mathrm{kgm}^{2}$ \\
\hline
\end{tabular}

\subsection{MID-based stabilizing controller design}

The critical delay may also be recovered using the MID property. The following result is a direct consequence of Theorem 4.2 from [9]. It provides a bound for the quasipolynomial roots' multiplicity. In addition, it explicitly computes the stabilizing MID-based controller's gains and delay.

Proposition 1 Considering equation (8), the following assertions hold.

i) The multiplicity of any given root of the quasipolynomial function (8) is bounded by 3 .

ii) For an arbitrary positive delay $\tau$, the quasipolynomial 8 admits a real spectral value at $s=s_{ \pm}$with algebraic multiplicity 3 if and only if,

$$
s_{ \pm}=\frac{-2 \pm \sqrt{2-\tau^{2} a_{0}}}{\tau},
$$

and the system parameters satisfy:

$$
\left\{\begin{array}{l}
b_{0}=\left(2 a_{0}+\frac{10 s_{ \pm}}{\tau}+\frac{6}{\tau^{2}}\right) \mathrm{e}^{s_{ \pm} \tau}, \\
b_{1}=\left(2 s_{ \pm}+\frac{2}{\tau}\right) \mathrm{e}^{s_{ \pm} \tau}
\end{array}\right.
$$

iii) If $\left(\star_{+}\right)$(respectively $\left.\left(\star_{-}\right)\right)$is satisfied then $s=s_{+}$is the spectral abscissa corresponding to [8) (respectively $s_{-}$cannot be the spectral abscissa corresponding to $(8)$ ). Furthermore, for an arbitrary delay $\tau$ the multiple spectral value at $s_{-}$ is always dominated by a single real root $s_{0}$.

iv) If $\left(\star_{+}\right)$is satisfied then the trivial solution is asymptotically stable if, and only if, $\tau \in] 0, \sqrt{-\frac{2}{a_{0}}}[$.

Thanks to the above result which is proved using the principle argument in [9], one exploits $\left(\star_{+}\right)$to assert that the maximal admissible multiplicity of the rightmost root at $s=0$ of $(8)$ is 3 . Next, (9) provides the critical feedback delay and the associated 
control gains

$$
\begin{aligned}
\tau_{\text {crit }} & =\sqrt{-\frac{2}{a_{0}}}, \\
k_{\mathrm{p}, \text { crit }} & =-\frac{2 I}{l} a_{0}, \\
k_{\mathrm{d}, \text { crit }} & =-\frac{2 I}{l} a_{0} \tau_{\text {crit }} .
\end{aligned}
$$

The corresponding numerical results are $\tau_{\text {crit }}=0.58 \mathrm{~s}, k_{\mathrm{p}, \text { crit }}=98.1 \mathrm{~N} / \mathrm{rad}, k_{\mathrm{d}, \text { crit }}=57.2 \mathrm{Ns} / \mathrm{rad}$. An alternative proof of the dominancy of the triple root at $s=0$ may be deduced following [5]. As a matter of fact, characteristic function may be rewritten as follows

$$
\begin{aligned}
D(s)=s^{2}+a_{0}+e^{-s \tau_{1}} & \left(-a_{0}-a_{0} \tau_{1} s\right) \\
& =s^{2}+a_{0}-a_{0} e^{-s \tau_{1}}\left(1+\tau_{1} s\right) \\
= & s^{2}\left(1+\int_{0}^{1} a_{0} \tau_{1}^{2} t e^{-s \tau_{1} t} \mathrm{~d} t\right) \\
& =s^{2}\left(1-\int_{0}^{1} 2 t e^{-s \tau_{1} t} \mathrm{~d} t\right) .
\end{aligned}
$$

In order to prove that there exists no root $s_{1}=\gamma_{1}+\mathrm{i} \omega_{1}$ of (19) such that $\gamma_{1}>0$, substitute $s_{1}$ into (19) which leads to

$$
\begin{aligned}
1=\left|\int_{0}^{1} 2 t e^{-s_{1} \tau_{1} t} \mathrm{~d} t\right| \leq \int_{0}^{1}\left|2 t e^{-s_{1} \tau_{1} t}\right| \mathrm{d} t & \\
& =\int_{0}^{1} 2 t e^{-\gamma_{1} \tau_{1} t} \mathrm{~d} t=: f\left(\gamma_{1}\right) .
\end{aligned}
$$

Observing that

$$
f\left(\gamma_{1}=0\right)=\int_{0}^{1} 2 t \mathrm{~d} t=1,
$$

it follows that for $\gamma_{1}>0$ the value of the integral is $f\left(\gamma_{1}\right)<1$ which proves the inconsistency of the hypothesis that the characteristic function (19) has an unstable root $s_{1}=\gamma_{1}+\mathrm{i} \omega_{1}$ with $\gamma_{1}>0$. Hence, no characteristic roots exist with positive real part, thus, the triple root $s=0$ is indeed the dominant root. 


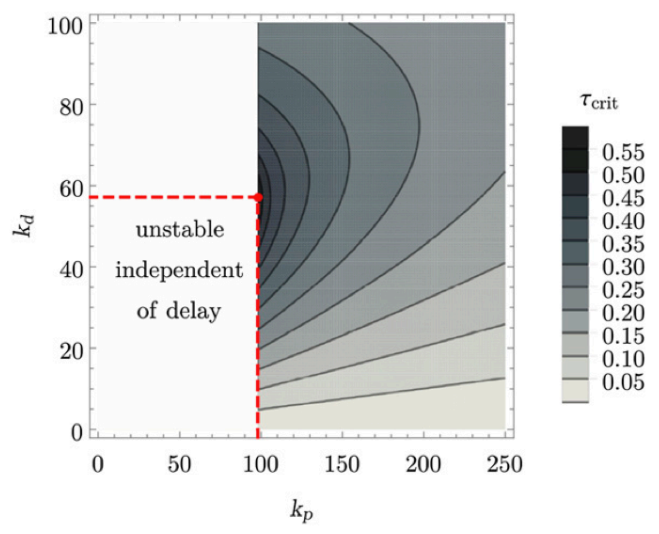

Figure 3: Critical delays for the single inverted pendulum.

\subsection{Critical delay by the WM method}

The WM method can also be applied to determine the critical delay (delay margin) for a fixed pair of control gains $\left(k_{\mathrm{p}}, k_{\mathrm{d}}\right)$. In this case 4 reads

$$
W\left(\omega^{2}\right)=\omega^{4}+\left(\frac{m g l}{I}-\frac{k_{\mathrm{d}}^{2} l^{2}}{4 I^{2}}\right) \omega^{2}+\left(\frac{m^{2} g^{2} l^{2}}{4 I^{2}}-\frac{k_{\mathrm{p}}^{2} l^{2}}{4 I^{2}}\right) .
$$

Only one positive root $\omega_{0}$ exists for any combination of the gains $k_{\mathrm{p}}$ and $k_{\mathrm{d}}$ and the critical delay is provided by 5 . The critical delays over the plane $\left(k_{\mathrm{p}}, k_{\mathrm{d}}\right)$ are shown in Fig. 3 where the maximal critical delay is attained by the critical gains $k_{\mathrm{p}, \mathrm{crit}}=98.1 \mathrm{~N} / \mathrm{rad}, k_{\mathrm{d}, \mathrm{crit}}=57.2 \mathrm{Ns} / \mathrm{rad}$.

\section{The double inverted pendulum}

The double inverted pendulum is often referred to as the most simple nonlinear multi-body system featuring all the properties of higher-degree-of-freedom nonlinear systems, such as complexity or chaos. As such, it is often used to demonstrate control concepts of complex systems [10, 16, 14]. The corresponding full state feedback involves the angular position and angular velocity of both pendulum segments, which implies that the number of control gains is four. Hence optimization and stabilization shall be performed in the four dimensional space of the control gains. Note that the double inverted pendulum is also an often used important model in human balancing research [25, 31, 23]. 


\subsection{Mechanical model}

The mechanical model of the double inverted pendulum is shown in Fig 4 The parameters of the model are $L_{1}=5 \mathrm{~m}, L_{2}=5 \mathrm{~m}, m_{1}=5 \mathrm{~kg}, m_{2}=5 \mathrm{~kg}$. The control force may be modeled as

$$
\begin{aligned}
F(t)= & k_{\mathrm{p} 1} \varphi_{1}(t-\tau)+k_{\mathrm{d} 1} \dot{\varphi}_{1}(t-\tau)+ \\
& k_{\mathrm{p} 2} \varphi_{2}(t-\tau)+k_{\mathrm{d} 2} \dot{\varphi}_{2}(t-\tau),
\end{aligned}
$$

where subscript 1 and 2 refer to the lower and upper pendulum, respectively. The linearized equation of motion reads

$$
\mathbf{M} \ddot{\mathbf{q}}(t)+\mathbf{S q}(t)=\mathbf{Q}(t),
$$

where the mass and the stiffness matrix are

$$
\begin{aligned}
\mathbf{M} & =\left(\begin{array}{ll}
1 & 0 \\
0 & 1
\end{array}\right) \\
\mathbf{S} & =\left(\begin{array}{cc}
-22.0725 & 4.4145 \\
13.2435 & -7.3575
\end{array}\right)
\end{aligned}
$$

and

$$
\mathbf{Q}(t)=\left(\begin{array}{c}
0 \\
F(t)
\end{array}\right) .
$$

\subsection{Stabilizability analysis}

Stability and stabilizability analysis of the double inverted pendulum is less straightforward compared to that of the single pendulum, since the orders of the plant and the controller are higher. Although D-curves can be generated in a similar way, the stable domains should be represented in the four-dimensional space $\left(k_{\mathrm{p} 1}, k_{\mathrm{p} 2}, k_{\mathrm{d} 1}, k_{\mathrm{d} 2}\right)$. In this section, stabilizability in terms of the critical delay is determined using the MID property and the results are confirmed with the numerical semidiscretization method.

First, the characteristic polynomial (1) with

$$
\begin{aligned}
& A(s)=s^{4}+a_{3} s^{3}+a_{2} s^{2}+a_{1} s+a_{0}, \\
& B(s)=b_{3} s^{3}+b_{2} s^{2}+b_{1} s+b_{0}
\end{aligned}
$$




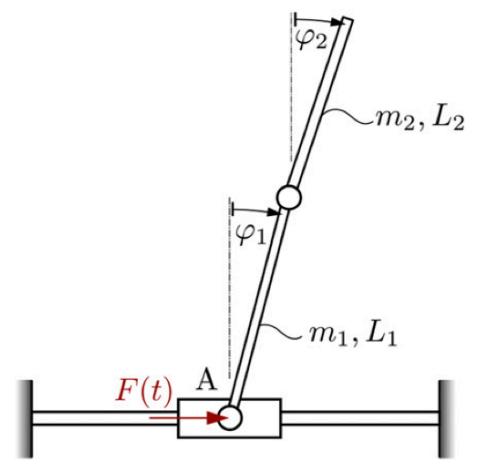

Figure 4: Double inverted pendulum

is investigated. The coefficients $a_{0}, a_{1}, a_{2}$ and $a_{3}$ are determined solely by the physical parameters $L_{1}, L_{2}, m_{1}, m_{2}$. The coefficients $b_{0}, b_{1}, b_{2}$ and $b_{3}$ of the delayed part of the quasipolynomial depend also on the control gains.

Now, we aim to find the control gains $k_{\mathrm{p} 1}, k_{\mathrm{p} 2}, k_{\mathrm{d} 1}$ and $k_{\mathrm{d} 2}$ (or equivalently, the parameters $b_{0}, b_{1}, b_{2}$ and $b_{3}$ ) that ensure the maximum feedback delay $\tau$. The maximal admissible multiplicity of the zero spectral value in this case is $2+4+3-1=8$. However, the number of unknown parameters being five $\left(b_{0}, b_{1}, b_{2}, b_{3}\right.$ and $\left.\tau\right)$, we cannot expect a multiplicity higher than five. As a matter of fact, zeroing the characteristic function and the first four derivatives yields

$$
\begin{gathered}
D(0)=a_{0}+b_{0}=0, \\
D^{\prime}(0)=a_{1}+b_{1}-b_{0} \tau=0, \\
D^{(\mathrm{II})}(0)=2 a_{2}+2 b_{2}-2 b_{1} \tau+b_{0} \tau^{2}=0,
\end{gathered}
$$




$$
\begin{gathered}
D^{(\mathrm{III})}(0)=6 a_{3}+6 b_{3}-6 b_{2} \tau+3 b_{1} \tau^{2}-b_{0} \tau^{3}=0, \\
D^{(\mathrm{IV})}(0)=24-24 b_{3} \tau+12 b_{2} \tau^{2}-4 b_{1} \tau^{3}+b_{0} \tau^{4}=0,
\end{gathered}
$$

so that zeroing the fifth derivative

$$
D^{(\mathrm{V})}(0)=-60 a_{3} \tau^{2}-40 a_{2} \tau^{3}-15 a_{1} \tau^{4}-4 a_{0} \tau^{5}=0
$$

is in contradiction with (34), which suggests that the maximal admissible multiplicity is 5. Solving $b_{0}, b_{1}, b_{2}$ and $b_{3}$ from 30 - 33 and substituting into 34 yields a polynomial in $\tau$. As in the previous section, the coefficients of the polynomial may be expressed in terms of the parameters of the plant as

$$
P(\tau)=24+24 a_{3} \tau+12 a_{2} \tau^{2}+4 a_{1} \tau^{3}+a_{0} \tau^{4}
$$

where

$$
\begin{gathered}
b_{0}=-a_{0}, \\
b_{1}=-a_{1}-a_{0} \tau \\
b_{2}=-\frac{1}{2}\left(2 a_{2}+2 a_{1} \tau+a_{0} \tau^{2}\right), \\
b_{3}=-\frac{1}{6}\left(6 a_{3}+6 a_{2} \tau+3 a_{1} \tau^{2}+a_{0} \tau^{3}\right) .
\end{gathered}
$$

The critical delay is the smallest positive $\tau$ solution of 36.

In the particular case of the double undamped inverted pendulum, the coefficients $a_{1}$ and $a_{3}$ are zero. For the sake of simplicity, we assume that the two pendulums are made of the same material, so their length is proportional to their mass. Without loss of generality, we consider the case where $m_{1}=\rho L_{1}$ and $m_{2}=\rho L_{2}$ with $\rho=1 \mathrm{~kg} / \mathrm{m}$. Consequently, the mass moment of inertia is $I_{1}=L_{1}^{3} / 12$ and $I_{2}=L_{2}^{3} / 12$ for the center of gravity of each pendulum. 
The non-zero coefficients of the characteristic equation are

$$
\begin{gathered}
a_{0}=\frac{9 g^{2}\left(L_{1}+2 L_{2}\right)}{L_{1}^{2} L_{2}}, \\
a_{2}=-\frac{3 g\left(L_{1}^{2}+7 L_{1} L_{2}+2 L_{2}^{2}\right)}{2 L_{1}^{2} L_{2}}, \\
b_{0}=-\frac{9 g\left(L_{1}+2 L_{2}\right)\left(k_{\mathrm{p} 1}+k_{\mathrm{p} 2}\right)}{L_{1}^{2} L_{2}\left(L_{1}+L_{2}\right)}, \\
b_{1}=-\frac{9\left(k_{\mathrm{d} 1}+k_{\mathrm{d} 2}\right) g\left(L_{1}+2 L_{2}\right)}{L_{1}^{2} L_{2}\left(L_{1}+L_{2}\right)}, \\
b_{2}=\frac{6 L_{1} L_{2} k_{\mathrm{p} 1}+3 L_{2}^{2} k_{\mathrm{p} 1}-3 L_{1}^{2} k_{\mathrm{p} 2}}{L_{1}^{2} L_{2}\left(L_{1}+L_{2}\right)}, \\
b_{3}=\frac{-3 k_{\mathrm{d} 2} L_{1}^{2}+3 k_{\mathrm{d} 1} l_{2}\left(2 L_{1}+L_{2}\right)}{L_{1}^{2} L_{2}\left(L_{1}+L_{2}\right)} .
\end{gathered}
$$

Note that for a physically realistic case $a_{0}>0$ and $a_{2}<0$. This property will be exploited later. The system is investigated with respect to the lengths $L_{1}$ and $L_{2}$ of the pendulum segments.

If $a_{1}=0$ and $a_{3}=0$ then the polynomial (36) reduces to an incomplete quadratic equation of the form

$$
P(\tau)=24+12 a_{2} \tau^{2}+a_{0} \tau^{4}=0 .
$$

Descartes' rule of signs indicates that the number of positive roots of 477) is two, hence, two positive and two negative solutions are obtained for $\tau$. The smaller positive value is the critical delay.

The two positive solutions for $\tau$ are

$$
\begin{gathered}
\tau_{1}=\sqrt{\frac{2\left(-3 a_{2}-\sqrt{3} \sqrt{3 a_{2}^{2}-2 a_{0}}\right)}{a_{0}},} \\
\tau_{2}=\sqrt{\frac{2\left(-3 a_{2}+\sqrt{3} \sqrt{3 a_{2}^{2}-2 a_{0}}\right)}{a_{0}} .}
\end{gathered}
$$


After substituting (41) and 42 , one can see that $3 a_{2}^{2}-2 a_{0}>0$, hence $\tau_{1}$ and $\tau_{2}$ are positive real roots indeed. The dominance of the quintuple root $s=0$ for the case $\tau=\tau_{1}<\tau_{2}$ is proved following the argument in [5].

If $\tau=\tau_{1}$ and the coefficients $b_{0}, b_{1}, b_{2}$ and $b_{3}$ satisfy equations (37), 38, , 39) and (40), respectively, then the characteristic function can be written in the following form:

$$
\begin{aligned}
D(s)= & s^{4}+a_{2} s^{2}+a_{0} \\
& -\frac{1}{6} e^{-s \tau_{1}}\left(6 a_{0}+6 a_{0} \tau_{1} s+\left(3 a_{0} \tau_{1}^{2}+6 a_{2}\right) s^{2}\right. \\
& \left.+\tau_{1}\left(a_{0} \tau_{1}^{2}+6 a_{2}\right) s^{3}\right) \\
= & s^{4}\left(1-\int_{0}^{1} e^{-s \tau_{1} t} \tau_{1}^{2} t\left(-a_{2}-\frac{1}{6} a_{0} \tau_{1}^{2} t^{2}\right) \mathrm{d} t\right) .
\end{aligned}
$$

To prove that there exists no root $s_{1}=\gamma_{1}+\mathrm{i} \omega_{1}$ of (50) such that $\gamma_{1}>0$, substitute $s_{1}$ into 50, one gets

$$
\begin{aligned}
1 & =\left|\int_{0}^{1} e^{-s_{1} \tau_{1} t} \tau_{1}^{2} t\left(-a_{2}-\frac{1}{6} a_{0} \tau_{1}^{2} t^{2}\right) \mathrm{d} t\right| \\
& \leq \int_{0}^{1} e^{-\gamma_{1} \tau_{1} t} \tau_{1}^{2} t\left|a_{2}+\frac{1}{6} a_{0} \tau_{1}^{2} t^{2}\right| \mathrm{d} t \\
& =-\int_{0}^{1} e^{-\gamma_{1} \tau_{1} t} \tau_{1}^{2} t\left(a_{2}+\frac{1}{6} a_{0} \tau_{1}^{2} t^{2}\right) \mathrm{d} t=: f\left(\gamma_{1}\right),
\end{aligned}
$$

since

$$
a_{2}+\frac{1}{6} a_{0} \tau_{1}^{2} t^{2}=a_{2}\left(1-t^{2}\right)-\frac{1}{\sqrt{3}} t^{2} \sqrt{3 a_{2}^{2}-2 a_{0}}<0 .
$$

For $\gamma_{1}=0$

$$
f\left(\gamma_{1}=0\right)=-\int_{0}^{1} \tau_{1}^{2} t\left(a_{2}+\frac{1}{6} a_{0} \tau_{1}^{2} t^{2}\right) \mathrm{d} t=1,
$$

so that for $\gamma_{1}>0$ the value of the integral is $f\left(\gamma_{1}\right)<1$ which proves the inconsistency of the hypothesis that the characteristic function 50 has an unstable root $s_{1}=\gamma_{1}+\mathrm{i} \omega_{1}$ with $\gamma_{1}>0$.

The critical delay obtained by the MID-based method over the plane $\left(L_{1}, L_{2}\right)$ is shown in Fig. 5 In order to verify the results numerically, the critical delay was determined by the semidiscretization method [15] combined with an interval halving technique [3] over a fixed grid of control parameters for $L_{1}=5 \mathrm{~m}$ and $L_{2}=5 \mathrm{~m}$. In this 


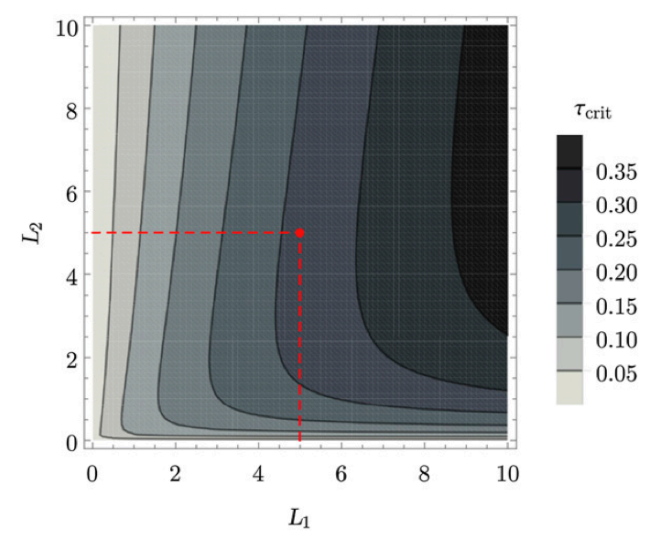

Figure 5: Critical delay for the double inverted pendulum deremined by the MID-based concept.

case, $\tau_{\text {crit }}=0.26 \mathrm{~s}$. A section of the stability regions in the four-dimensional space of the control gains is represented in Fig. 6 for $\tau=0.25 \mathrm{~s}$. As can be seen, the stable region disappears indeed at the critical parameters.

\section{Conclusion}

The MID property is employed to determine the critical feedback delay for the stabilization of a double inverted pendulum with full delayed state feedback. The method was shown to determine the critical delay with significantly smaller computational effort compared to other numerical methods, e.g., the Walton-Marschall or the semidiscretization method for a series of control gains combination. The main benefit of applying the MID-based approach is that the control space does not have to be swept, since the control gains associated with the critical delay are derived in closed form. Furthermore, in order to get the critical delay only the zeros of a quasipolynomial should be determined, while the corresponding control gains can be calculated by solving a finite set of linear equations. This is a useful feature especially for higher order plants or controllers design.

To the best knowledge of the authors, similar analytical method to determine the critical delay for higher-order system such as the double inverted pendulum is not available in the literature yet. The method applied in this paper to the double inverted 

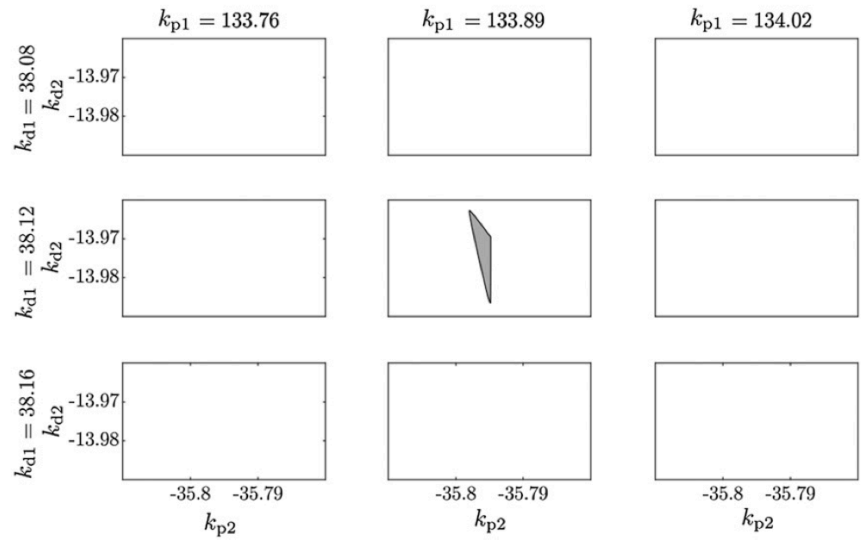

Figure 6: Stability diagrams for the double inverted pendulum with $L_{1}=L_{2}=5 \mathrm{~m}$ in the neighbourhood of the critical point when $\tau=0.25 \mathrm{~s}$.

pendulum can be adopted to more general systems under certain conditions on the characteristic function, see, e.g. [9].

\section{Acknowledgements}

The research reported in this paper was supported by the Hungarian-French Bilateral Scientific and Technological Cooperation Fund under Grant no. 2017-2.2.5TÉT-FR-2017-00007, by the Higher Education Excellence Program of the Ministry of Human Capacities in the frame of Biotechnology research area of Budapest University of Technology and Economics (BME FIKP-BIO), by the Hungarian-Chinese Bilateral Scientific and Technological Cooperation Fund under Grant no. 2018-2.1.14TÉT-CN-2018-00008, by the National Research, Development and Innovation Fund (TUDFO/51757/2019-ITM, Thematic Excellence Program and by the ÚNKP-19-3 New National Excellence Program of the Ministry for Innovation and Technology). IB is partially financially supported by a public grant overseen by the French National Research Agency (ANR) as part of the "Investissement d'Avenir" program, through the "iCODE Institute project" funded by the IDEX Paris-Saclay and a grant from Hubert Curien (PHC) BALATON, project number 40502NM. 


\section{References}

[1] Ackermann J (2002) Robust Control - The Parameter Space Approach. SpringerVerlag, London, UK

[2] Atay FM (1999) Balancing the inverted pendulum using position feedback. Applied Mathematics Letters 12(5):51-56

[3] Bachrathy D, Stépán G (2012) Bisection method in higher dimensions and the efficiency number. Periodica Polytechnica Mechanical Engineering 56(2):81-86

[4] Boussaada I, Niculescu SI (2018) On the dominancy of multiple spectral values for time-delay systems with applications. IFAC-PapersOnLine 51(14):55-60

[5] Boussaada I, Unal HU, Niculescu SI (2016) Multiplicity and Stable Varieties of Time-delay Systems: A Missing Link. In: 22nd International Symposium on Mathematical Theory of Networks and Systems (MTNS), Minneapolis, United States

[6] Boussaada I, Niculescu SI, Tliba S, Vyhlídal T (2017) On the coalescence of spectral values and its effect on the stability of time-delay systems: Application to active vibration control. Procedia IUTAM 22(Supplement C):75 - 82

[7] Boussaada I, Niculescu SI, Trabelsi K (2018) Toward a decay rate assignment based design for time-delay systems with multiple spectral values. In: Proceeding of the 23rd International Symposium on Mathematical Theory of Networks and Systems, pp 864-871

[8] Boussaada I, Tliba S, Niculescu SI, Ünal H, Vyhlídal T (2018) Further remarks on the effect of multiple spectral values on the dynamics of time-delay systems. application to the control of a mechanical system. Linear Algebra and its Applications 542:589-604

[9] Boussaada I, Niculescu SI, El-Ati A, Perez-Ramos R, Trabelsi K (2020) Multiplicity-induced-dominancy in parametric second-order delay differential 
equations: analysis and application in control design. To appear in ESAIM: COCV pp 1-32

[10] Casanova V, Salt J, Piza R, Cuenca A (2012) Controlling the Double Rotary Inverted Pendulum with Multiple Feedback Delays. International Journal of Computers Communications \& Control 7(1):20-38

[11] Ciezkowski M (2016) Method for determination of interaction between a twowheeled self-balancing vehicle and its rider. Mechanics 22(5):416-424

[12] Habib G, Miklós A, Enikov ET, Stépán G, Rega G (2017) Nonlinear model-based parameter estimation and stability analysis of an aero-pendulum subject to digital delayed control. International Journal of Dynamics and Control 5(3):629-643

[13] Hayes ND (1950) Roots of the transcendental equation associated with a certain difference-differential equation. Journal of the London Mathematical Society s125(3):226-232

[14] He C, Huang K, Chen X, Zhang Y, Zhao H (2018) Transportation control of cooperative double-wheel inverted pendulum robots adopting Udwadia-control approach. Nonlinear Dynamics 91(4):2789-2802

[15] Insperger T, Stepan G (2011) Semi-discretization for time-delay systems. Springer, New York

[16] Lukowska A, Tomaszuk P, Ciezkowski M, Dzierzek K, Recko M (2018) Acceleration control approach of double inverted pendulum system. In: 2018 19th International Carpathian Control Conference (ICCC), pp 122-127

[17] Ma D, Chen J (2018) Delay margin of low-order systems achievable by PID controllers. IEEE Transactions on Automatic Control 64(5):1958-1973

[18] Maurer C, Peterka RJ (2005) A new interpretation of spontaneous sway measures based on a simple model of human postural control. Journal of Neurophysiology 93(1):189-200 
[19] Michiels W, Niculescu SI (2007) Stability and stabilization of time delay systems - An eigenvalue based approach. SIAM Publications, Philadelphia

[20] Milton J, Insperger T (2019) Acting together, destabilizing influences can stabilize human balance. Philosophical Transactions of the Royal Society A 377(2153):20180,126

[21] Milton J, Cabrera JL, Ohira T, Tajima S, Tonosaki Y, Eurich CW, Campbell SA (2009) The time-delayed inverted pendulum: implications for human balance control. Chaos: An Interdisciplinary Journal of Nonlinear Science 19(2):026110

[22] Molnar Tamas G T Insperger (2016) On the robust stabilizability of unstable systems with feedback delay by finite spectrum assignment. Journal of Vibration and Control 22(3):649-661

[23] Morasso P, Cherif A, Zenzeri J (2019) Quiet standing: The single inverted pendulum model is not so bad after all. PloS ONE 14(3):e0213,870

[24] Olgac N, Sipahi R (2002) An exact method for the stability analysis of timedelayed linear time-invariant (LTI) systems. IEEE Transactions on Automatic Control 47(5):793-797

[25] Pinter BJ, van Swigchem R, van Soest AJK, Rozendaal LA (2008) The dynamics of postural sway cannot be captured using a one-segment unverted pendulum model: A PCA on segment rotations during unperturbed stance. Journal of Neurophysiology 100:3197-3208

[26] Qin ZC, Li X, Zhong S, Sun JQ (2014) Control experiments on time-delayed dynamical systems. Journal of Vibration and Control 20(6):827-837

[27] Sieber J, Krauskopf B (2004) Bifurcation analysis of an inverted pendulum with delayed feedback control near a triple-zero eigenvalue singularity. Nonlinearity 17(1):85-103

[28] Sieber J, Krauskopf B (2005) Extending the permissible control loop latency for the controlled inverted pendulum. Dynamical Systems 20(2):189-199 
[29] Stepan G (1989) Retarded dynamical systems. Longman, Harlow

[30] Stepan G (2009) Delay effects in the human sensory system during balancing. Philosophical Transactions of the Royal Society A 367:1195-1212

[31] Suzuki Y, Nomura T, Casadio M, Morasso P (2012) Intermittent control with ankle, hip, and mixed strategies during quiet standing: A theoretical proposal based on a double inverted pendulum model. Journal of Theoretical Biology 310:55-79

[32] Walton K, Marshall JE (1987) Direct method for TDS stability analysis. IEE Proceedings D-Control Theory and Applications 134(2):101-107

[33] Xu Q, Stepan G, Wang Z (2017) Balancing a wheeled inverted pendulum with a single accelerometer in the presence of time delay. Journal of Vibration and Control 23(4):604-614

[34] Zhu J, Qi T, Ma D, Chen J (2018) Limits of Stability and Stabilization of TimeDelay Systems. Springer 